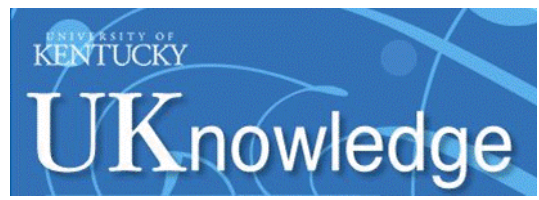

University of Kentucky

UKnowledge

Electrical and Computer Engineering Faculty

Publications

Electrical and Computer Engineering

4-19-2018

\title{
Effective Room-Temperature Ammonia-Sensitive Composite Sensor Based on Graphene Nanoplates and PANI
}

\author{
Zongbiao Ye \\ University of Electronic Science and Technology of China, China \\ Yan Chen \\ Chengdu University of Information Technology, China \\ Bohao Liu \\ University of Electronic Science and Technology of China, China \\ Yuanjie Su \\ University of Electronic Science and Technology of China, China \\ Zhi Chen \\ University of Kentucky, zhi.chen@uky.edu
}

See next page for additional authors

Follow this and additional works at: https://uknowledge.uky.edu/ece_facpub

Part of the Electrical and Computer Engineering Commons

Right click to open a feedback form in a new tab to let us know how this document benefits you.

\section{Repository Citation}

Ye, Zongbiao; Chen, Yan; Liu, Bohao; Su, Yuanjie; Chen, Zhi; Tai, Huiling; and Jiang, Yadong, "Effective Room-Temperature Ammonia-Sensitive Composite Sensor Based on Graphene Nanoplates and PANI" (2018). Electrical and Computer Engineering Faculty Publications. 25.

https://uknowledge.uky.edu/ece_facpub/25

This Article is brought to you for free and open access by the Electrical and Computer Engineering at UKnowledge. It has been accepted for inclusion in Electrical and Computer Engineering Faculty Publications by an authorized administrator of UKnowledge. For more information, please contact UKnowledge@lsv.uky.edu. 


\section{Effective Room-Temperature Ammonia-Sensitive Composite Sensor Based on Graphene Nanoplates and PANI}

Digital Object Identifier (DOI)

https://doi.org/10.1149/2.0201807jss

Notes/Citation Information

Published in ECS Journal of Solid State Science and Technology, v. 7, issue 7, p. Q3148-Q3152.

(C) The Author(s) 2018. Published by ECS.

This is an open access article distributed under the terms of the Creative Commons Attribution 4.0 License (CC BY, http://creativecommons.org/licenses/by/4.0/), which permits unrestricted reuse of the work in any medium, provided the original work is properly cited.

Authors

Zongbiao Ye, Yan Chen, Bohao Liu, Yuanjie Su, Zhi Chen, Huiling Tai, and Yadong Jiang 


\title{
Effective Room-Temperature Ammonia-Sensitive Composite Sensor Based on Graphene Nanoplates and PANI
}

\author{
Zongbiao Ye, ${ }^{1}$ Yan Chen, ${ }^{2}$ Bohao Liu, ${ }^{1}$ Yuanjie Su, ${ }^{1}$ Zhi Chen, ${ }^{3, z}$ Huiling Tai, ${ }^{1, z}$ \\ and Yadong Jiang ${ }^{1}$ \\ ${ }^{1}$ State Key Laboratory of Electronic Thin Films and Integrated Devices, School of Optoelectronic Information, \\ University of Electronic Science and Technology of China, Chengdu, Sichuan 610054, People's Republic of China \\ ${ }^{2}$ Sichuan Province Key Laboratory of Information Materials and Devices Application, College of Optoelectronic \\ Technology, Chengdu University of Information Technology, Chengdu 610225, People's Republic of China \\ ${ }^{3}$ Department of Electrical \& Computer Engineering and Center for Nanoscale Science \& Engineering, University of \\ Kentucky, Lexington, Kentucky 40506, USA
}

\begin{abstract}
The graphene nanoplate (GN)-polyaniline (PANI) composite was developed via in-situ polymerization method and simultaneously assembled on interdigital electrodes (IDEs) at low temperature for ammonia $\left(\mathrm{NH}_{3}\right)$ detection. The assembled composite sensor showed excellent sensing performance toward different concentrations of $\mathrm{NH}_{3}, 1.5$ of response value and $123 \mathrm{~s} / 204 \mathrm{~s}$ for the response/recovery time to $15 \mathrm{ppm} \mathrm{NH}_{3}$. Meanwhile, an interesting supersaturation phenomenon was observed at high concentration of $\mathrm{NH}_{3}$. A reasonable speculation was proposed for this special sensing behavior and the mechanism for enhanced sensing properties was also analyzed.

(c) The Author(s) 2018. Published by ECS. This is an open access article distributed under the terms of the Creative Commons Attribution 4.0 License (CC BY, http://creativecommons.org/licenses/by/4.0/), which permits unrestricted reuse of the work in any medium, provided the original work is properly cited. [DOI: 10.1149/2.0201807jss]

(c)) BY
\end{abstract}

Manuscript submitted February 14, 2018; revised manuscript received March 26, 2018. Published April 19, 2018. This paper is part of the JSS Focus Issue on Semiconductor-Based Sensors for Application to Vapors, Chemicals, Biological Species, and Medical Diagnosis.

Among the toxic gases of the interest, ammonia is a prominent example for its wide applications in industrial manufacturing and daily life. ${ }^{1}$ It can even be generally produced in natural processes in animals, human and plants. ${ }^{2}$ Ammonia can irritates skin, eyes and respiratory tract of humans when the concentration reaches to a certain value (the safety threshold is $\sim 25 \mathrm{ppm}$ in air). ${ }^{3}$ It is also flammable at concentration of ca. $15 \%-28 \%$ by volume in air. ${ }^{4}$ Therefore, many approaches have been employed to detect ammonia, including gas chromatography, ${ }^{5}$ polarography, ${ }^{6}$ fluorometry ${ }^{7}$ and spectrophotometry. ${ }^{8}$ Meanwhile, with the consideration of the need for cheap, fast and efficient sensors, semiconductor-based gas sensors have been developed quickly. ${ }^{9}$

As an emerging 2-D material, graphene has attracted much attention worldwide for its large specific surface area ${ }^{10}$ and excellent electrical properties. ${ }^{11,12}$ However, no material can be satisfactorily applied in any field, especially in complex scenarios. Theoretical and experimental studies have shown graphene performs limited selectivity to different kinds of gas species. ${ }^{13}$ Composition with other functional materials would be an expectable choice. The conducting polymer, especially polyaniline (PANI),${ }^{14}$ is a promising choice, as their low cost and ability for room-temperature detection. ${ }^{15}$ Extensive studies reported that the composite of PANI and graphene demonstrates efficient charge transport and collection, ${ }^{16}$ as well as enhanced thermal and chemical stability. ${ }^{17}$

Herein, GN-PANI nanocomposite film was synthesized by in-situ chemical oxidative polymerization of aniline in a functional graphene nanoplate (GN) suspension, and was simultaneously assembled onto a substrate with interdigital electrodes (IDEs) at low temperature. It suggests that the composite film sensor shows enhanced $\pi$ electrons conjugation system, large specific surface area and stronger intermolecular interaction. Benefit from this, the composite showed a much improved sensing performance comparing with bare GN and bare PANI based sensors.

\section{Experimental}

Materials._Aniline ( $\geq 99.5 \%$ ), poly (diallyldimenthylammonium chloride) solution (PDDA) and poly (sodium 4-styrenesulfonate) (PSS) were purchased from Sigma-Aldrich Co., USA. Ammonium persulfate (APS) and hydrochloric acid $(\mathrm{HCl})$ were obtained from Chengdu Kelong Chemical Reagent Co., China. Graphene nanoplates were purchased from Nanjing XFNANO Materials Tech Co., Ltd.

Sensor fabrication.-The IDEs was pre-treated with polycationic PDDA aqueous solution (1\%) and polyanionic PSS aqueous solution $(2 \mathrm{mg} / \mathrm{mL}, \mathrm{PH} \approx 1$, adjusted by $\mathrm{HCl}$ ) in sequence to get a negatively charged layer on the surface and increase the hydrophily of substrate before use. Subsequently, the GN-PANI nanocomposite thin film was formed on the IDEs by in-situ self-assembly method at $\sim 12^{\circ} \mathrm{C}$. Specifically, $1 \mathrm{~mL}$ aniline was dissolved in $10 \mathrm{~mL} \mathrm{HCl} \mathrm{(2} \mathrm{M)} \mathrm{solution.} \mathrm{After}$ dissolving, $1.5 \mathrm{mg}$ GN was added into the solution under vigorous stirring, then $10 \mathrm{~mL} 0.1 \mathrm{M}$ APS hydrochloric acid solution was gradually dropped into the mixture within 2 mins. With the introduction of APS in the mixture solution, aniline was chemically driven to polymerize into PANI, the polymerization process could be depicted as Figure 1. When the color of solution turns to pale blue, the pre-treated IDEs substrate was immersed into the solution, as shown as Figure 2. After 20 mins polymerization process, the IDEs with GN-PANI nanocomposite film was removed from the black blue solution and washed with diluted $\mathrm{HCl}$ solution. At last, the device was dried at room temperature for a whole night. For comparison, a pure PANI film sensor was prepared using the same process without addition of GN. The GN film was prepared by spray method using GN suspension solution.

\section{Results and Discussion}

The dynamic temperature and illustration of preparation processing of polymerization process of PANI and GN was recorded as shown as Figure 2. The entire process was kept below $14^{\circ} \mathrm{C}$. With the ongoing polymerization of aniline, the temperature of the reaction solution increased quickly, finally it reach a relative stable situation at $13.5^{\circ} \mathrm{C}$. It shows that the reaction is a typical exothermic. The reaction 

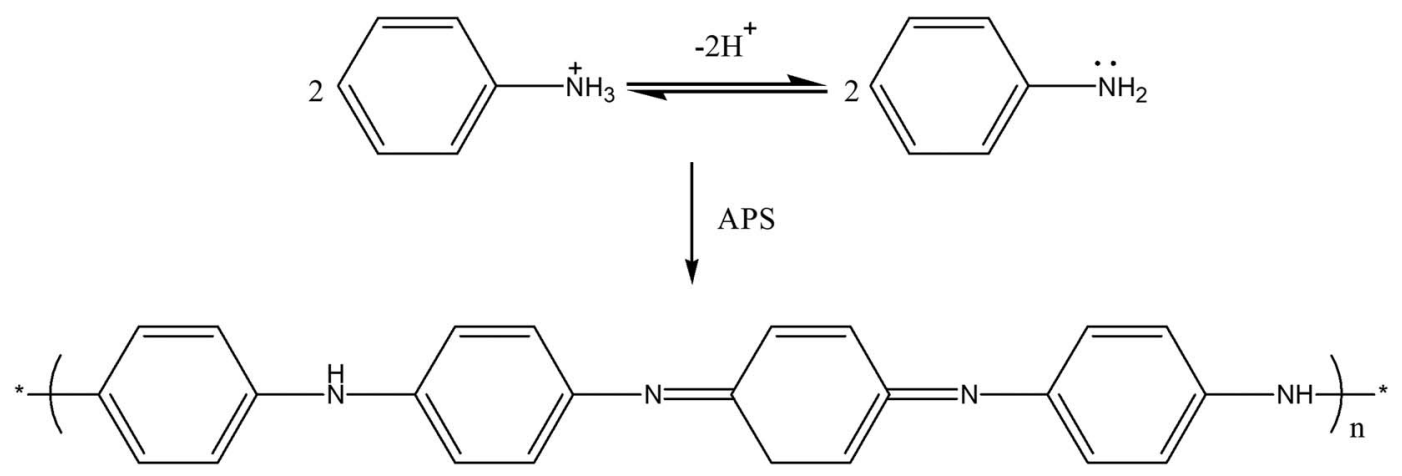

Figure 1. Reaction equation graph of polymerization process of PANI.

process can be concluded that the aniline monomer can be ionized and adsorb on the surface of GN, then under the oxidation of APS, PANI chains can be formed and thin film would be convolved on the IDEs.

The UV-vis spectra of GN, PANI and GN-PANI composite thin film were depicted in Figure 3. There is no other obvious absorption peak can be observed except the typical peak around $260 \mathrm{~nm}$ due to $\pi$-conjugation system. ${ }^{18}$ For PANI sample, the peak shows at high wavelength $748 \mathrm{~nm}-760 \mathrm{~nm}$, could be attributed to quinoid excitation and $\pi$-polaron transition. ${ }^{19}$ A short peak at $328 \mathrm{~nm}$ could be attributed to the $\pi-\pi^{*}$ transition of the benzenoid ring. ${ }^{17}$ These peaks are typical for doped PANI emeraldine salt. ${ }^{20}$ The GN-PANI thin film shows similar curve shape to that of $\mathrm{HCl}$ doped PANI, except that the absorbance intensity of the former was higher than the latter. The two peaks that mentioned before can also be observed in the composite film. However, the peak at $748 \mathrm{~nm}-760 \mathrm{~nm}$ has shifted to $593 \mathrm{~nm}-611 \mathrm{~nm}$ contrast to that of PANI. In addition, the composite film shows a new peak at $285 \mathrm{~nm}$, it could be attributed to the introduction of GN. ${ }^{21}$ Moreover, it has a redshift with comparison to GN. All the results indicate that with the addition of GN in PANI, the intermolecular interaction between two materials was improved ${ }^{22}$ and conducting system of PANI and GN was delocalized, ${ }^{23}$ conjugated $\pi$-system was enhanced ${ }^{19}$ and the energy bandgap would be widened as a result. ${ }^{24}$

As shown in introduction, even if a trace amount of $\mathrm{NH}_{3}$ inhaled is actually toxic in people's daily life. Therefore, monitoring $\mathrm{NH}_{3}$ concentration level plays an important role in human health. To estimate the sensing performance of sensors, response (S) is intro-

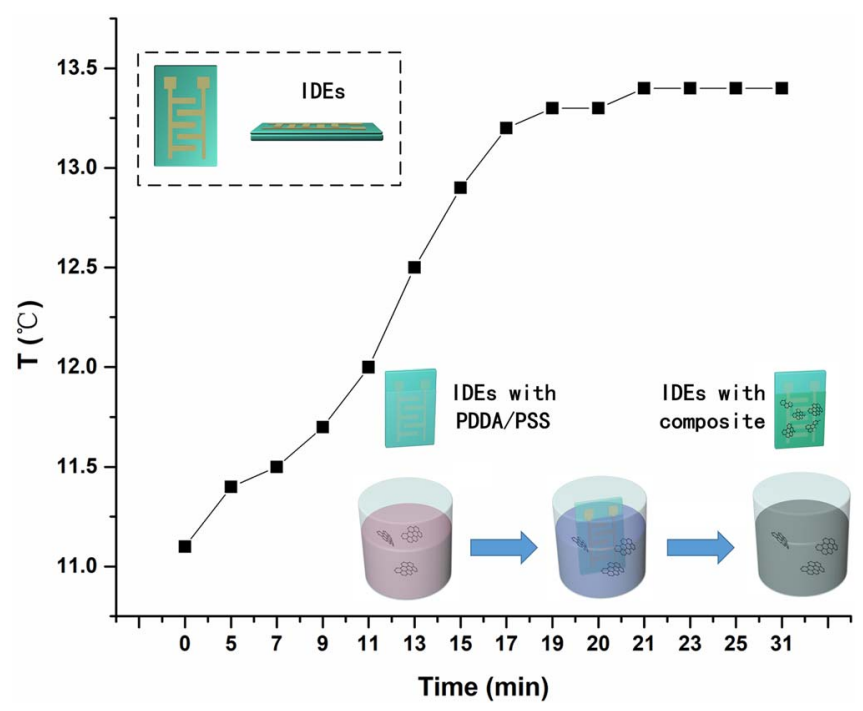

Figure 2. The temperature change of the reaction solution (the inset shows the illustraton of IDEs and polymerization process of composite film). duced to evaluate sensitive behavior of sensors, which can be defined as $S=\Delta R / R_{\text {origin }}$, where $\Delta R$ means the resistance change before and after exposure to $\mathrm{NH}_{3}$, and $\mathrm{R}_{\text {origin }}$ represents the resistance before exposure to target gas. The response time and recovery time are defined as the sensor achieves $90 \%$ change of total resistance variation.

The sensing responses of the sensors were measured under room temperature. Figure 4 displays the dynamic response of sensors based on GN, PANI and GN-PANI composite film toward $12.5 \mathrm{ppm}, 20 \mathrm{ppm}$, $25 \mathrm{ppm}, 30 \mathrm{ppm}, 35 \mathrm{ppm}$ and $100 \mathrm{ppm}$ respectively. The resistance immediately increases when the sensor was exposed in $\mathrm{NH}_{3}$. When exposure to air, the resistance of sensors decreased significantly and gradually restore to base state. The resistance increase-decrease process could be explained by the doping-undoping process as shown as Figure 5. Ammonia could be ionized when adsorbed on the surface of PANI film, and hole traps would be produced. The decrease of positive charged carries density in acid-doping PANI backbone, such as solitons, polarons and bipolarons ${ }^{25}$ could be caused by the formation of ammonium cations, which leads to the partial reduction of the device conductance. Accordingly, the acid doped PANI nanofibers switched from the emeraldine salt state (conductive state) to emeraldine base state (insulating state) gradually, resulting in increase of the resistance as shown in Figure 4. When $\mathrm{NH}_{3}$ was withdrew from the test chamber, $\mathrm{NH}_{3}$ molecule and proton could be liberated and released from $\mathrm{NH}_{4}{ }^{+}$and insulating base state could restore to conducting salt state. Then the resistance of PANI based sensors recovered to its original conducting state accordingly.

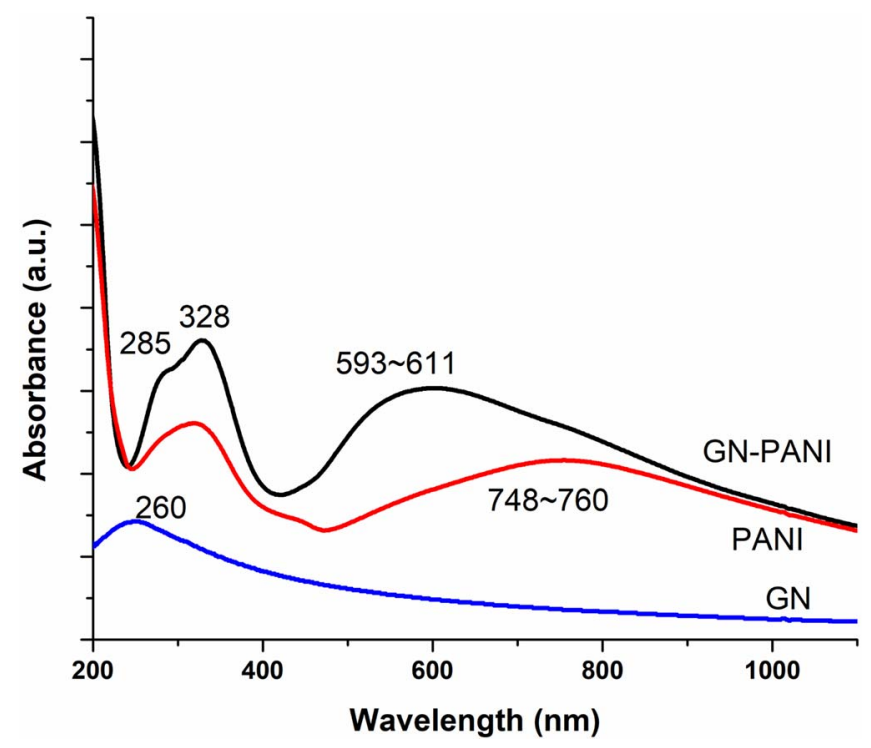

Figure 3. UV-vis of spectra of GN, PANI and GN-PANI composite. 


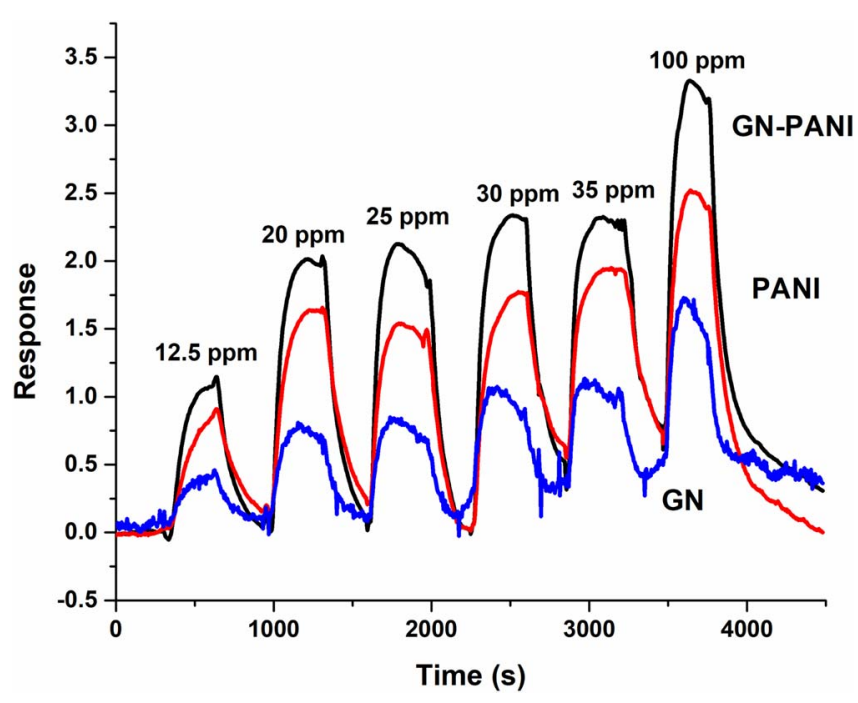

Figure 4. Response curve of GN, PANI and GN-PANI composite under exposure to different concentration levels of ammonia gas.

As increase of the concentration of $\mathrm{NH}_{3}$ gas, the resistances of devices significantly increase and evidently achieve the saturation state. The GN based sensor could not recover to its initial resistance under no $\mathrm{NH}_{3}$ injection and performs baseline drift in a varying degree. The drift would be more serious under higher concentration of $\mathrm{NH}_{3}$. For PANI based sensor, it shows rapid response and recovery process. When exposing to high concentration of $\mathrm{NH}_{3}(>30 \mathrm{ppm})$, the sensor requires more time to restore to its original resistance. With a certain time, the sensor could recover to its initial state even under exposure to $100 \mathrm{ppm}$ of $\mathrm{NH}_{3}$. For GN-PANI composite sensor, it shows enhanced response in comparison with GN and PANI based sensors. The response under exposure to $100 \mathrm{ppm}$ is 3.3 for GN-PANI composite, 2.5 for PANI and 1.4 for GN based sensor, respectively.

There is an interesting response-recovery phenomenon appeared at $100 \mathrm{ppm}$, the resistances of devices increase significantly and achieve saturation state quickly after exposure to $\mathrm{NH}_{3}$, then there is a remarkable ramp-down phase showed up during the "supersaturation state". To investigate this situation, we executed measurement of these sensors from high concentration of $\mathrm{NH}_{3}$ to low concentration (150 ppm to $50 \mathrm{ppm}$ ) as shown in Figure 6 . We can observe clearly that under $50 \mathrm{ppm}$ exposure to $\mathrm{NH}_{3}$, the sensors still keep a flat saturation state as shown in Figure 4, and under $100 \mathrm{ppm}$ exposure to $\mathrm{NH}_{3}$, the sensors showed a lightly decline. However, for the sensors exposure to $150 \mathrm{ppm} \mathrm{NH}$, the decline degree become more serious, which is in agreement with other reports. ${ }^{26}$ An earlier study suggested that the chemisorbed $\mathrm{H}_{2}$ can react with oxygen from the environment to produce $\mathrm{H}_{2} \mathrm{O}$ and the presence of $\mathrm{H}_{2} \mathrm{O}$ ruins the ability of PANI to sense

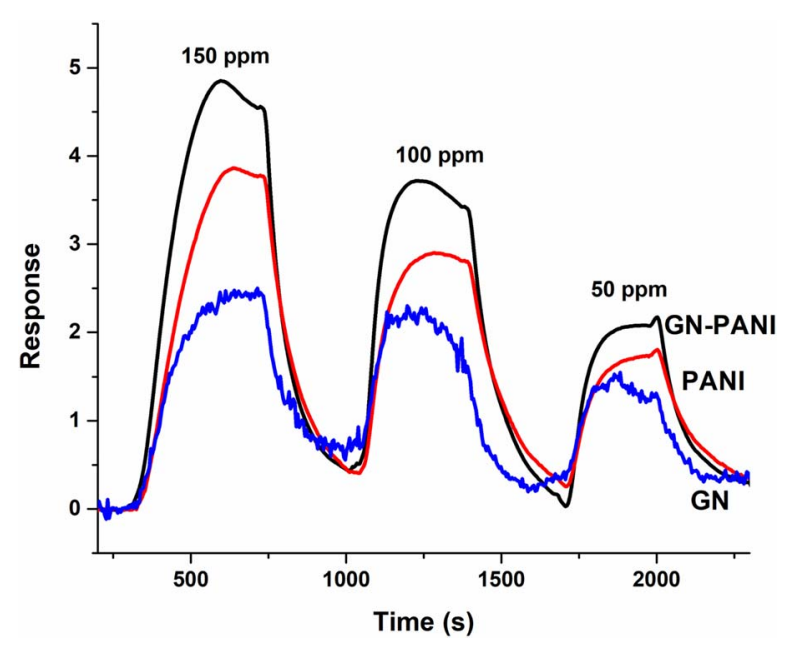

Figure 6. Response curve of GN, PANI and GN-PANI composite under exposure to high concentration level of ammonia gas.

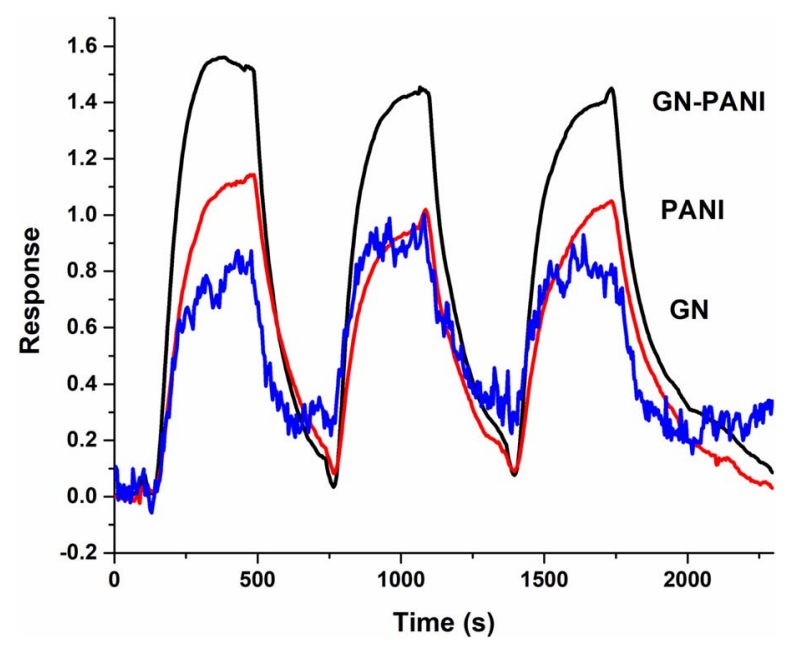

Figure 7. Repetitive response curve of GN, PANI and GN-PANI composite under exposure to $15 \mathrm{ppm}$ of ammonia gas.

$\mathrm{H}_{2} \cdot{ }^{27,28}$ Analogously, we attribute this phenomenon to the production of $\mathrm{H}_{2} \mathrm{O}$ when exposure to high concentration of $\mathrm{NH}_{3}$. The presence of $\mathrm{H}_{2} \mathrm{O}$ would cover the adsorption sites on the surface of thin-film sensor and decrease the adsorption quantity of $\mathrm{NH}_{3}$ molecules. On the other hand, the emerging $\mathrm{H}_{2} \mathrm{O}$, due to strong dissolving capacity to $\mathrm{H}_{2} \mathrm{O}$, would capture the doped protonic acid $(\mathrm{HCl})$ from the PANI chains, which equals to a de-doping process of PANI. As a result,

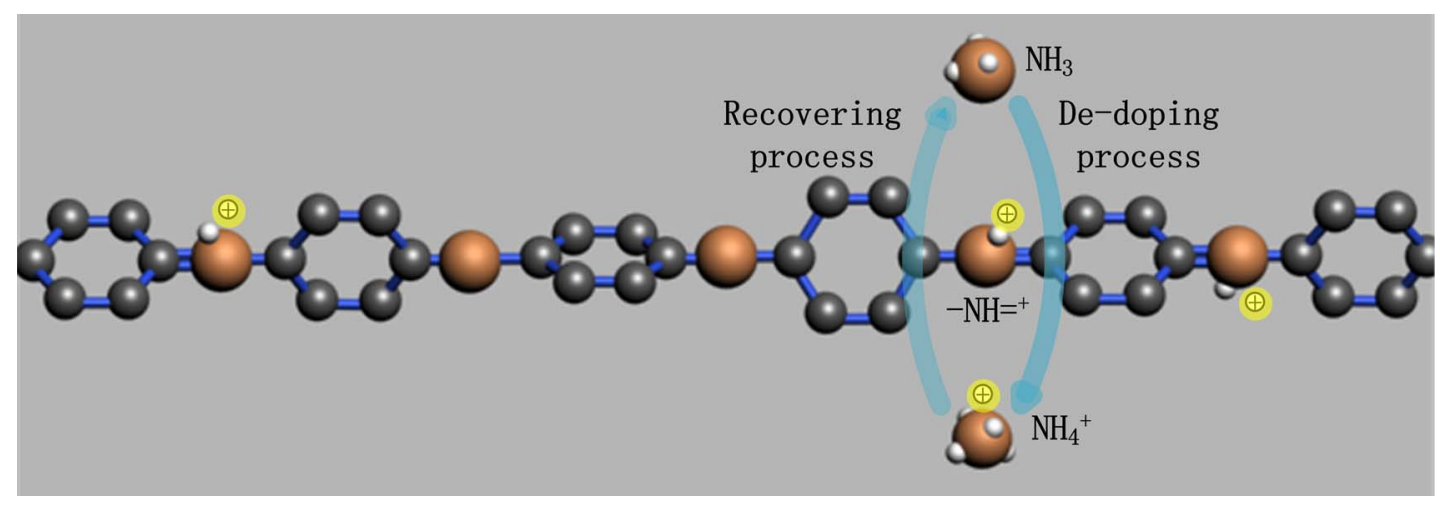

Figure 5. Schematic illustration of doping and de-doping process of PANI chain 

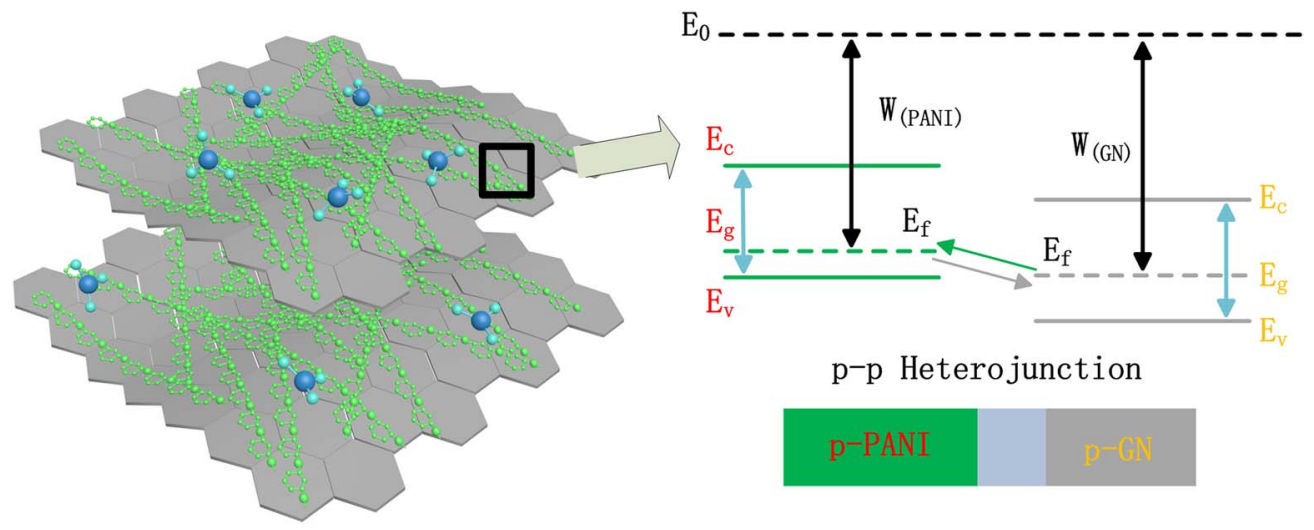

$\mathrm{p}-\mathrm{p}$ Heterojunction

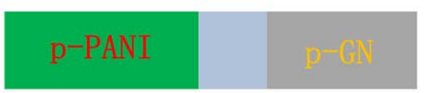

Figure 8. Schematic illustration of proposed sensing mechanism of GN-PANI composite.

the sensors would suffer a saturation-degradation process. The resistance decreases significantly during the supersaturation period when exposure to high concentration of $\mathrm{NH}_{3}$ ( $\geq 100 \mathrm{ppm}$ ).

In addition, sensors based on GN, PANI and GN-PANI composite show good repeatability to three exposure cycles of $15 \mathrm{ppm}$ $\mathrm{NH}_{3}$ as shown in Figure 7. The GN and PANI show a different extent of fluctuation in response-recovery process during 3 cycles. The composite shows improved repeatability in comparison to GN and PANI based sensors (the response is 1.54/1.45/1.47 for composite, 1.13/1.01/1.04 for PANI and 0.86/1.00/0.92 for GN). Meanwhile, the response/recovery time can be extracted from the first sensing cycle, it is $123 \mathrm{~s} / 204 \mathrm{~s}$ for composite, $172 \mathrm{~s} / 246 \mathrm{~s}$ for PANI and $153 \mathrm{~s} / 241 \mathrm{~s}$ for GN, respectively. Apparently, the composite sensor shows enhanced sensing properties. However, under the consideration of humidity effect, all the sensors including the composite sensor were susceptible to moisture. Therefore, the stability of hybrid sensor in the humidity atmosphere is still of concern for $\mathrm{NH}_{3}$ sensing, and further work needs to be implemented.

The improved $\mathrm{NH}_{3}$ sensing performance of composite sensor could be assigned to synergistic effect of PANI and GN. A large specific surface area (as shown in Figure 8) due to that the PANI nanoparticles and chains are anchored on the surface of GN, is benefit to the adsorption of $\mathrm{NH}_{3}$ gas on the composite surface and the deprotonation at the interface of the composite. ${ }^{29}$ Consequently, the response-recovery speed would be enhanced. In addition, the GN provides high conductivity and carriers mobility, and the electron transfer energy between PANI and GN is relatively lower $(\sim 101 \mathrm{meV}){ }^{30}$ Therefore, the activation energy and enthalpy of adsorption by heterojunction (as shown in Figure 8) for $\mathrm{NH}_{3}$ would be reduced. ${ }^{31}$ Meanwhile, $\pi$ electrons cloud of PANI and GN could be overlapped and form a $\pi-\pi$ conjugation system for charge interaction between $\mathrm{NH} 3$ gas molecules and $\pi$ electrons, which means the composite owns more $\pi$ electrons cloud area than that of GN and PANI. Therefore, the carrier interaction and transportation of composite would be improved, and consequently increases the sensing performance. However, the speculation has not been verified precisely, detailed understanding for the role of GNPANI network in the sensing mechanism of the composite still needs further investigation.

\section{Conclusions}

A resistance-type sensor based on GN-PANI was successfully prepared by in-situ chemical oxidative polymerization of aniline in GN suspension and simultaneously deposited on IDEs. The composite sensor shows a better sensing performance than that of GN and PANI based sensors due to synergistic effect of PANI and GN. Besides, supersaturation phenomenon occurring at high concentration of $\mathrm{NH}_{3}$ was observed and investigated. It reveals that the composite sensor is simple, inexpensive, sensitive and room-temperature reliable, which is expected to be built in hand-held optoelectronic devices for further functional convergence.

\section{Acknowledgment}

This work is supported by the Creative Research Groups Award of National Nature Science Foundations of China (grant No. 61421002) and National Natural Science Foundation of China (grant No. 61671115 and No.51707015).

\section{ORCID}

Zhi Chen (1) https://orcid.org/0000-0002-4451-5626

\section{References}

1. G. K. Mani and J. B. B. Rayappan, "A highly selective room temperature ammonia sensor using spray deposited zinc oxide thin film," Sens. Actuators, B, 183, 459 (2013).

2. J. Pearson and G. R. Stewart, "The deposition of atmospheric ammonia and its effects on plants," New Phytol. 125(2), 283 (1993).

3. B. Timmer, W. Olthuis, and A. Van Den Berg, "Ammonia sensors and their applications-a review," Sens. Actuators, B, 107(2), 666 (2005).

4. K. P. Yoo, K. H. Kwon, N. K. Min, M. J. Lee, and C. J. Lee, "Effects of $\mathrm{O}_{2}$ plasma treatment on $\mathrm{NH}_{3}$ sensing characteristics of multiwall carbon nanotube/polyaniline composite films," Sens. Actuators, B, 143(1), 333 (2009).

5. R. R. Reston and E. S. Kolesar, "Silicon-micromachined gas chromatography system used to separate and detect ammonia and nitrogen dioxide. I. Design, fabrication, and integration of the gas chromatography system," J. Microelectromech. Syst., 3(4), 134 (1994).

6. T. Osakai, T. Kakutani, and M. Senda, "A novel amperometric ammonia sensor," Anal. Sci., 3(6), 521 (1987).

7. M. A. Arnold and T. J. Ostler, "Fiber optic ammonia gas sensing probe," Anal. Chem., 58(6), 1137 (1986).

8. P. Caglar and R. Narayanaswamy, "Ammonia-sensitive fibre optic probe utilising an immobilised spectrophotometric indicator," Analyst. 112(9), 1285 (1987).

9. F. Fang, L. Bai, H. Sun, Y. Kuang, X. Sun, T. Shi, D. Song, P. Guo, H. Yang, Z. Zhang, Y. Wang, J. Luo, and J. Zhu, "Hierarchically porous indium oxide nanolamellas with ten-parts-per-billion-level formaldehyde-sensing performance," Sens. Actuators, B, 206, 714 (2015).

10. K. M. Mohsin, A. Srivastava, M. S. Fahad, and M. A. Khan, "Quantum Capacitance of Hybrid Graphene Copper Nanoribbon." ECS J. Solid State Sci. Technol., 6(10), M133 (2017).

11. A. C. Neto, F. Guinea, N. M. Peres, K. S. Novoselov, and A. K. Geim, "The electronic properties of graphene," Rev. Mod. Phys., 81(1), 109 (2009).

12. A. K. Geim and K. S. Novoselov, "The rise of graphene," Nat. Mater., 6(3), 183 (2007).

13. G. Lu, S. Park, K. Yu, R. S. Ruoff, L. E. Ocola, D. Rosenmann, and J. Chen, "Toward practical gas sensing with highly reduced graphene oxide: a new signal processing method to circumvent run-to-run and device-to-device variations," ACS nano, 5(2), 1154 (2011).

14. X. Liu, W. Yang, H. Xu, and W. Wang, "Pulse Electropolymerization and Thermoelectrical Performances of Carbon Nanotubes/Polyaniline Composite Film," ECS J. Solid State Sci. Technol., 5(5), M27 (2016).

15. Q. Hao, H. Wang, X. Yang, L. Lu, and X. Wang, "Morphology-controlled fabrication of sulfonated graphene/polyaniline nanocomposites by liquid/liquid interfacial polymerization and investigation of their electrochemical properties," Nano Res. 4(4), 323 (2011). 
16. X. Huang, N. Hu, R. Gao, Y. Yu, Y. Wang, Z. Yang, E. S. Kong, H. Wei, and Y. Zhang, "Reduced graphene oxide-polyaniline hybrid: preparation, characterization and its applications for ammonia gas sensing," J. Mater. Chem., 22(42), 22488 (2012).

17. Y. Guo, T. Wang, F. Chen, X. Sun, X. Li, Z. Yu, P. Wan, and X. Chen, "Hierarchical graphene-polyaniline nanocomposite films for high-performance flexible electronic gas sensors," Nanoscale, 8(23), 12073 (2016).

18. K. Sheng, H. Bai, Y. Sun, C. Li, and G. Shi, "Layer-by-layer assembly of graphene/polyaniline multilayer films and their application for electrochromic devices," Polym. 52(24), 5567 (2011).

19. X. M. Feng, R. M. Li, Y. W. Ma, R. F. Chen, N. E. Shi, Q. L. Fan, and W. Huang, "One-step electrochemical synthesis of graphene/polyaniline composite film and its applications," Adv. Funct. Mater., 21(15), 2989 (2011).

20. Q. Wu, Y. Xu, Z. Yao, A. Liu, and G. Shi, "Supercapacitors based on flexible graphene/polyaniline nanofiber composite films," ACS nano. 4(4), 1963 (2010).

21. Z. Ye, Y. Jiang, H. Tai, N. Guo, G. Xie, and Z. Yuan, "The investigation of reduced graphene oxide@ $\mathrm{SnO}_{2}$-polyaniline composite thin films for ammonia detection at room temperature," J. Mater. Sci. - Mater. Electron., 26(2), 833 (2015).

22. H. Bai, K. Sheng, P. Zhang, C. Li, and G. Shi, "Graphene oxide/conducting polymer composite hydrogels," J. Mater. Chem., 21(46), 18653 (2011).

23. F. Gu, S. F. Wang, C. F. Song, M. K. Lü, Y. X. Qi, G. J. Zhou, D. Xu, and D. R. Yuan, "Synthesis and luminescence properties of $\mathrm{SnO}_{2}$ nanoparticles," Chem. Phys. Lett., 372(3-4). 451 (2003).
24. Z. Wu, X. Chen, S. Zhu, Z. Zhou, Y. Yao, W. Quan, and B. Liu, "Enhanced sensitivity of ammonia sensor using graphene/polyaniline nanocomposite," Sens. Actuators, B, 178, 485 (2013).

25. M. O. Ansari, M. M. Khan, S. A. Ansari, I. Amal, J. Lee, and M. H. Cho, "Enhanced thermoelectric performance and ammonia sensing properties of sulfonated polyaniline/graphene thin films," Mater. Lett., 114, 159 (2014).

26. H. Tai, Y. Jiang, G. Xie, J. Yu, X. Chen, and Z. Ying, "Influence of polymerization temperature on $\mathrm{NH}_{3}$ response of $\mathrm{PANI} / \mathrm{TiO}_{2}$ thin film gas sensor," Sens. Actuators, $B, \mathbf{1 2 9}(1), 319$ (2008).

27. J. D. Fowler, S. Virji, R. B. Kaner, and B. H. Weiller, "Hydrogen detection by polyaniline nanofibers on gold and platinum electrodes," J. Phys. Chem. C, 113(16), 6444 (2009).

28. L. Al-Mashat, K. Shin, K. Kalantar-zadeh, J. D. Plessis, S. H. Han, R. W. Kojima, R. W. Kaner, D. Li, X. Gou, S. J. Ippolito, and W. Wlodarski, "Graphene/polyaniline nanocomposite for hydrogen sensing," J. Phys. Chem. C, 114(39), 16168 (2010).

29. S. Bai, Y. Zhao, J. Sun, Y. Tian, R. Luo, D. Li, and A. Chen, "Ultrasensitive room temperature $\mathrm{NH}_{3}$ sensor based on a graphene-polyaniline hybrid loaded on PET thin film," Chem. Commun., 51(35), 7524 (2015).

30. R. G. Amorim, A. Fazzio, A. J. da Silva, and A. R. Rocha, "Confinement effects and why carbon nanotube bundles can work as gas sensors," Nanoscale, 5(7), 2798 (2013).

31. B. P. de Lacy Costello, P. Evans, R. J. Ewen, C. L. Honeybourne, and N. M. Ratcliffe, "Novel composite organic-inorganic semiconductor sensors for the quantitative detection of target organic vapours," J. Mater. Chem., 6(3), 289 (1996). 